\title{
Image guider subsystem analysis for the GHAPS project
}

\author{
Michael Lewis, Jeffrey Juergens, Eliot Aretskin-Hariton \\ NASA John H. Glenn Research Center, Cleveland, Ohio \\ Robert Woodruff \\ Robert Woodruff Consulting, Boulder, Colorado
}

\begin{abstract}
The Gondola for High-Altitude Planetary Science (GHAPS) project is a balloon-borne astronomical observatory designed operate in the UV, Visible, and near-mid IR spectral region. The GHAPS Optical Telescope Assembly (OTA) is designed around a one meter aperture narrow field-of-view telescope with near-diffractionlimited performance. GHAPS will utilize Wallops Arc-Second Pointing System (WASP) for pointing the OTA with an accuracy of 1 arc second or better. WASP relies heavily on a self-contained star tracker assembly to determine the OTA line of sight. Preliminary structural analysis indicates that potential misalignments could be present between the OTA line of sight and the star tracker FOV center during the expected flight conditions that could compromise GHAPS pointing accuracy.

In response the GHAPS project undertook a trade study to resolve the following issues: (1) estimate the worst case long-term (or bias) pointing misalignments for the GHAPS opto-mechanical configuration, (2) examine the need for additional hardware to correct pointing errors, and (3) determine the best hardware and software implementation to do so. Quantitative comparisons of performance and qualitative estimates of other factors such as mass, volume, power consumption, and cost are combined into an overall assessment of potential solutions. Results are discussed and a recommended implementation is given that is optimized to best achieve pointing performance goals, while minimizing impact to the design, cost, and resources of the GHAPS project.
\end{abstract}

\section{INTRODUCTION}

The Gondola for High-Altitude Planetary Science (GHAPS) project is a balloon-borne astronomical observatory designed to answer critical questions identified in NASA's Planetary Science Decadal Survey. GHAPS builds on previous balloon missions, expanding the offering for planetary scientists to include highly capable long duration missions. GHAPS is designed for a minimum of five flights, each up to 100 days in duration, minimizing maintenance between flights. A competitive process will be used to select investigators based primarily on proposed science.

GHAPS optical performance will be optimized for observations in the $300 \mathrm{~nm}$ to $5 \mu \mathrm{m}$ wavelength region covering UV, visible, and near-mid IR. GHAPS is being designed around a one meter aperture narrow-field-of-view telescope with a sub-arc second pointing accuracy. Utilizing NASA's stratospheric balloon capabilities, GHAPS will fly at altitudes between 30 to $40 \mathrm{~km}$ where astrophysical and planetary observations can be made above more than $99.5 \%$ of Earth's atmospheric water vapor and other atmospheric disturbances.

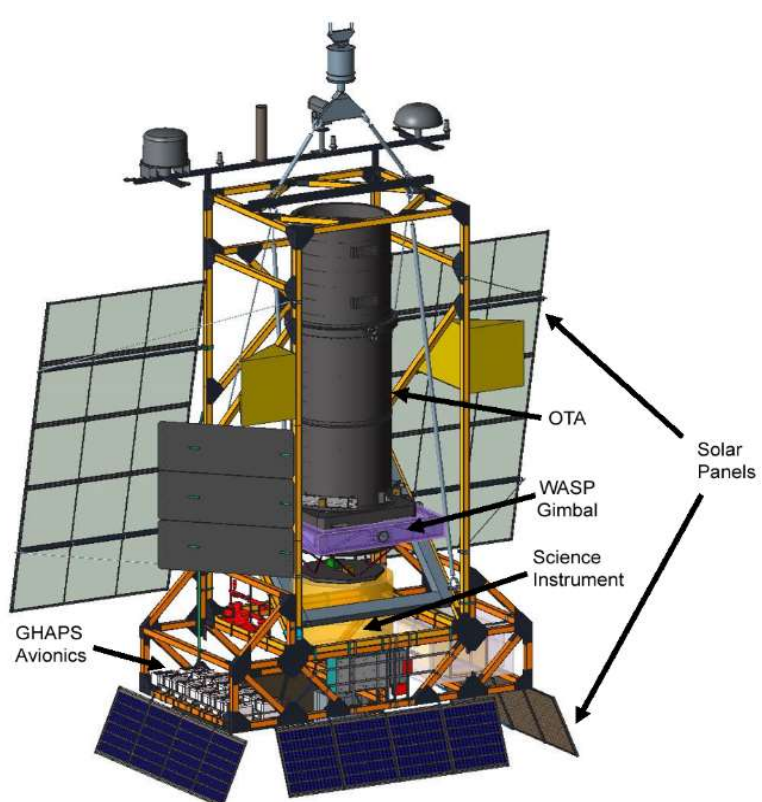

Figure 1. GHAPS Gondola Assembly Concept 
The GHAPS Gondola includes the Optical Telescope Assembly (OTA), the Wallops Arc-Second Pointing System (WASP), avionics, thermal, power system, and the Gondola structure and software. Figure 1 show the current GHAPS Gondola Assembly. The primary structure and the subsystems are required to be strong and capable of being disassembled for transport, survive landing, and light-weighted to the extent possible to allow for highly capable long duration flights.

\subsection{Trade Study Background}

GHAPS will utilize the WASP pointing system which combines the information from multiple sensors, including a high precision star tracker which is primarily responsible for achieving the desired pointing accuracy of 1 arc sec. or better. The star tracker is mounted outboard of GHAPS OTA and utilizes its own lens system and image sensor. Preliminary analysis of the OTA stiffness reveled that a potential misalignment between OTA line-of sight (LOS) and star tracker LOS could arise due to diurnal thermal effects and OTA gravity-induced deflection over the range of possible elevation angles. This misalignment has the potential to significantly offset (also referred to as bias offset) the OTA's FOV with respect to the desired science target FOV, as directed by the WASP pointing system. As a result the project initiated a trade study to address the following issues:

1) Determine worst case long-term (bias) misalignments that could develop between OTA LOS and WASP/Star tracker LOS.

2) Determine if additional hardware is needed to reduce this misalignment to comply with the project goal of lower than 1 arc sec. pointing accuracy.

3) If additional hardware capability is needed, determine the best implementation for GHAPS.

\section{WORST CASE OTA AND STAR TRACKER MISALIGNMENT}

At the onset of the trade study, the best information on thermal and gravity deflections of the OTA was found in the OTA Preliminary Design Review data package. The package contained estimated deflections of the primary and secondary mirrors as a function of elevation angle and thermal conditions. Using decenter and tip/tilt sensitives derived from the optical model of the GHAPS OTA, deflections were then incorporated into a simple model that converted the OTA primary and secondary deflections into changes in LOS. The result is shown in Figure 2 for elevation-induced misalignments and thermal effects are shown in Figure 3.

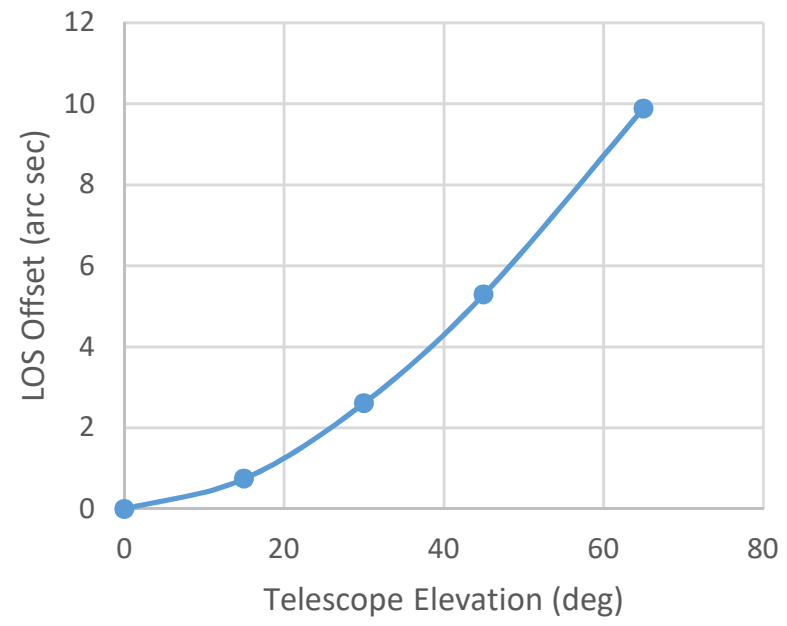

Figure 2 Worst Case OTA Gravity Induced LOS Bias Error.

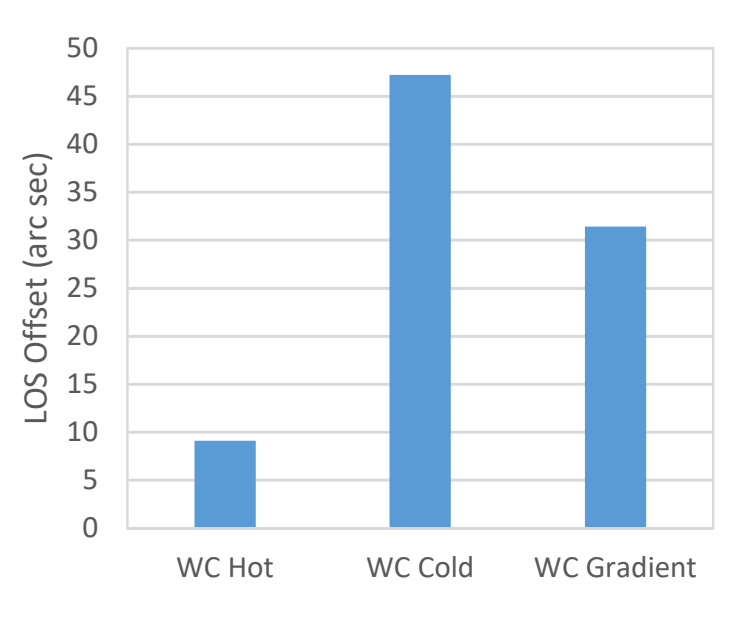

Figure 3. Worst Case Thermal Induced Bias Error. 
As is evident, the worst case misalignment is well beyond the $1 \mathrm{arc}$ sec. desired pointing accuracy. This estimate does not include deflections and misalignment that the Star tracker and lens will undergo in the same conditions. For the purpose of this worst case analysis, the star tracker is assumed to be a rigid body whose LOS is unaffected by either elevation angle or thermal environment.

Ground-based OTA and Star tracker boresight alignment can be performed at a median position, both thermally and in terms of elevation angle, which reduces the LOS misalignment with respect to the OTA's average LOS, but maximum LOS excursions are still well above the $1 \mathrm{arc} \mathrm{sec}$. goal. More elaborate calibration schemes were examined where the OTA and Star tracker misalignment are characterized at intervals over the entire range of telescope elevations and thermal environments. Ultimately, this approach was deemed too costly in terms of resources, facilities, and schedule by the project. A simpler, lower resource, alternative would be to add LOS offset detection to the OTA in the form of an OTA guide camera. This camera would reside near the OTA focal plane and image a portion of the OTA FOV. Using stars detected in the OTA Guide Camera FOV, the OTA FOV center in celestial coordinates can be determined to a very high accuracy. The OTA FOV center coordinates can then be provided to the WASP pointing system to properly offset LOS misalignments between Star

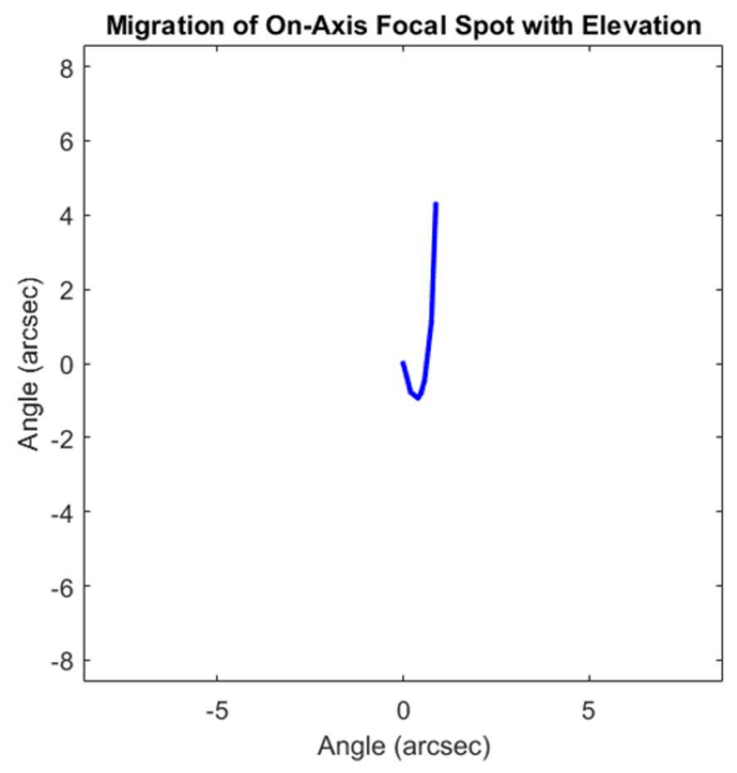

Figure 4. OTA Gravity Induced Pointing Errors Over Full Elevation Range
Tracker and the OTA.

At this stage of the trade study the worst case LOS misalignments between OTA and Star Tracker LOS had been shown to significantly exceed the pointing accuracy goals, and it appears that the best approach to solving the issue will require additional OTA capability in the form of a Facility Guide Camera dedicated to detecting the OTA LOS.

At a later point in the trade study, a more refined analysis of the OTA pointing performance was presented which showed marked improvement in gravity and thermal induced pointing errors. Results are shown in Figure 4. The maximum in-flight pointing error due to gravity is approximately 6.0 arc sec. Similarly, the thermal effects are expected to be less than 2.0 arc sec./hr. Although the most recent estimates are much better in terms of maximum expected bias error, the analysis still assumes perfect mechanical joints and material properties. Perhaps additional design iterations could improve pointing further, but until it can be shown, with margin, in a high fidelity model, the project should proceed assuming some form of pointing bias measurement and correction is needed from either the GHAPS facility camera or the science instrument.

\section{FACILITY GUIDE CAMERA TRADE}

Many factors will influence the optimum guide camera implementation, however in all cases the camera will need to image very faint stars with an acceptable signal to noise ratio, due to the relatively small FOV that it can utilize. Figure 5 shows the relevant FOVs enabled by the GHAPS overall configuration when incorporating a guide camera. The Wave Front Sensor (WFS) is a separate system that is used by GHAPS to reduce OTA wave front error in flight, and this portion of the FOV cannot be utilized by the guide camera. The Science FOV is area of the OTA focal plane that most Science Instruments are expected to utilize, and cannot be used by the Facility Guide System (FGS) as well. Therefore the maximum FOV available to the FGS is the OTA's full 450 arc sec. diameter FOV minus the Science FOV, and minus the WFS FOV.

\subsection{Photometry}

As is apparent from the small FOV available to the FGS, it will need the capability to image relatively dim stars (approx. > visible magnitude 16) for a high probability of even a single star capture over any random section of the 
entire sky. To adequately predict the FGS limiting star magnitude a first order photometric model was developed that included the GHAPS OTA, sky background, star radiance, FGS Image Sensor, and any reimaging optics that may be needed.

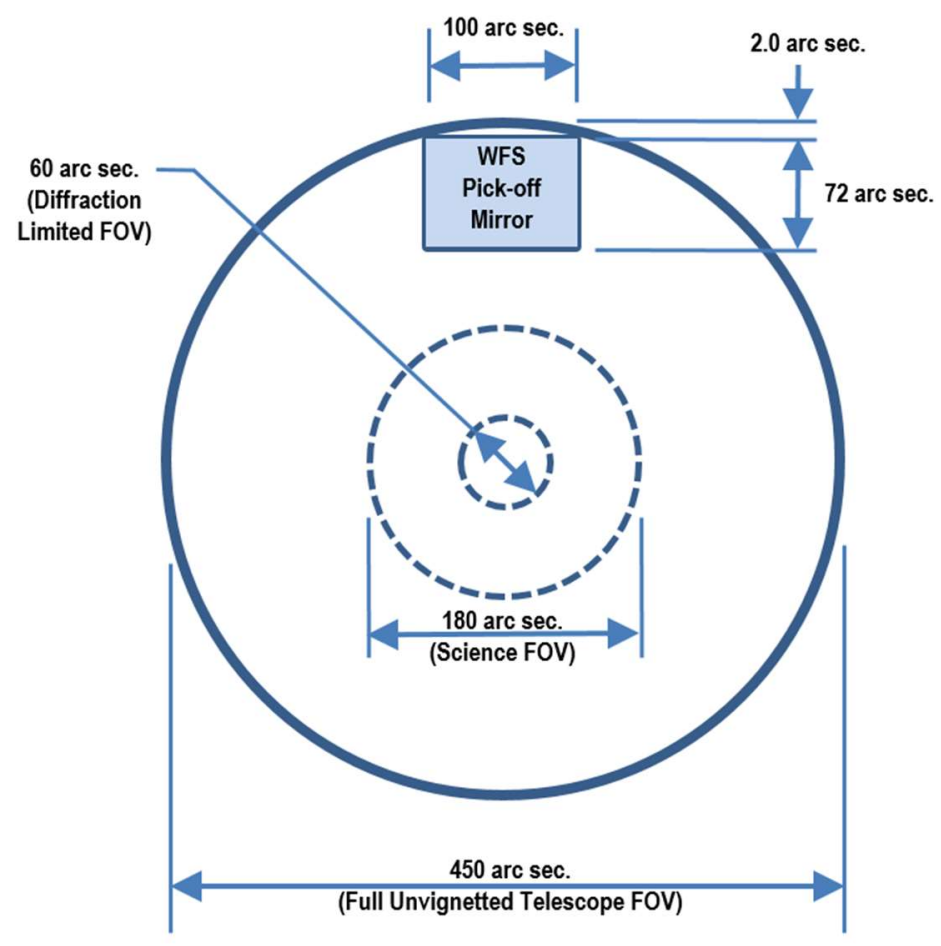

Figure 5. GHAPS OTA FOV at Cassegrain Focus

\subsubsection{Image Sensors}

The GHAPS OTA design goal is for nearly diffraction-limited image quality from visible to Mid-IR (0.5 to 5 um). Potential FGS image sensors could operate anywhere in this spectral range. For the trade study the following types of image sensor technologies were examined in terms of performance: 1) Scientific CMOS sCMOS), 2) Depletion CCD (D-CCD), 3) InGaAs, 4) HgCdTe 5) InSb, 6) e-APD (HgCdTe).

Both image sensor and camera performance data were obtained from various manufactures, and candidates with the best compromise in sensitivity, noise, size, weight, and power was chosen for each technology.

shows the relevant characteristics for each of the sensor technologies. A number of the sensor technologies require closed cycle sterling coolers (cryocooler) to achieve good noise figures. Cryocoolers add mass, volume, and power consumption compared to devices that only require thermoelectric cooling (TE). The GHAPS OTA and Instrument Bay will be susceptible to random and sinusoidal vibration, which is inherent with all cryocooler devices, hence image sensor technologies that require a cryocooler were removed from further consideration.

Table 1. FGS Image Sensor Technologies

\begin{tabular}{|c|c|c|c|c|c|}
\hline Sensor & QE/Band & Size & Noise & Camera Mfg. & Physical \\
\hline sCMOS & $\begin{array}{l}\text { 80-95\% Peak } \\
(0.4-1.0 u m) \\
\text { Peak@0.6um }\end{array}$ & $\begin{array}{l}\text { Up to } 4 \mathrm{~K} \times 4 \mathrm{~K} \\
\text { Pixel: } 4.5-13 \mathrm{um}\end{array}$ & Excellent & $\begin{array}{l}\text { Photometric } \\
\text { Andor, Ximea Raptor, } \\
\text { etc. }\end{array}$ & 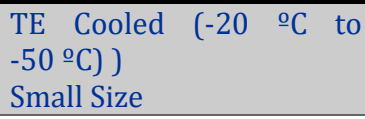 \\
\hline $\begin{array}{l}\text { Depletion- } \\
\text { CCD }\end{array}$ & $\begin{array}{l}\text { 70-95\% Peak } \\
\text { (0.5-1.0um) } \\
\text { Peak@0.8um }\end{array}$ & $\begin{array}{l}\text { Up to } 1 \mathrm{~K} \times 1 \mathrm{~K} \\
\text { Pixel: } 13-20 \mathrm{um}\end{array}$ & Good & $\begin{array}{l}\text { Princeton Imaging, } \\
\text { FLI }\end{array}$ & $\begin{array}{l}\text { TE Cooled }\left(-60^{\circ} \mathrm{C}\right) \\
\text { Small Size }\end{array}$ \\
\hline InGaAs & $\begin{array}{l}80-90 \% \text { Peak } \\
(0.5-1.7 \text { um }) \\
\text { Peak@1.2um }\end{array}$ & $\begin{array}{l}\text { Up to } 1 \mathrm{~K} \times 1 \mathrm{~K} \\
\text { Pixel: } 13-20 \mathrm{um}\end{array}$ & Good-Fair & $\begin{array}{l}\text { PI, FLIR, IR Cameras, } \\
\text { Raptor, etc. }\end{array}$ & $\begin{array}{llll}\text { TE cooled } & (-20 & \stackrel{\circ}{ } \mathrm{C} & \text { to } \\
-50 & \stackrel{\circ}{ } \mathrm{C} & & \\
\text { Small to Med Size } & & \end{array}$ \\
\hline $\begin{array}{l}\text { HgCdTe } \\
\text { (MCT) }\end{array}$ & $\begin{array}{l}\text { 80-90\% Peak } \\
(1.6-5.3 \text { um) } \\
\text { Custom Peak }\end{array}$ & $\begin{array}{l}\text { Up to } 4 \mathrm{~K} \times 4 \mathrm{~K} \\
\text { Pixel: } 10-18 \mathrm{um}\end{array}$ & Good & $\begin{array}{l}\text { Low Res- FLIR } \\
\text { High Res }- \text { Teledyne }\end{array}$ & $\begin{array}{l}\text { Sterling Cooler }\left(120^{\circ} \mathrm{K} \text { to }\right. \\
\left.77^{\circ} \mathrm{K}\right) \\
\text { Larger Size }\end{array}$ \\
\hline A-MCT & $\begin{array}{l}\text { 70\% QE Peak } \\
(1.1-2.5 \text { um })\end{array}$ & $\begin{array}{l}320 \times 256 \\
\text { Pixel: } 24 u m\end{array}$ & Excellent & $\begin{array}{l}\text { First Light, Imaging, } \\
\text { Selex, Voxtel }\end{array}$ & $\begin{array}{l}\text { Sterling Cooler }\left(77^{\circ} \mathrm{K}\right) \\
\text { Larger size }\end{array}$ \\
\hline $\mathrm{InSb}$ & $\begin{array}{l}\text { 80-90\% Peak } \\
\text { (1-5.5um) Flat }\end{array}$ & $\begin{array}{l}\text { Up to } 1 \mathrm{~K} \times 1 \mathrm{~K} \\
\text { Pixel: } 12-20 \mathrm{um}\end{array}$ & Good-Fair & FLIR, L3, Raytheon & $\begin{array}{l}\text { Sterling Cooler }\left(77^{\circ} \mathrm{K}\right) \\
\text { Larger size }\end{array}$ \\
\hline
\end{tabular}


Depletion-CCD (D-CCD) technology does not require a cryocooler, however there are other operational characteristics that can make it less desirable. D-CCD devices are standard CCD structures that are first back thinned and have an addition layer of silicon added which tunes their spectral response toward the longer wavelengths, compared to bulk silicon spectral response. This process opens the device up to interference filter-like effects due to the differences in index of refraction. The resulting effect is a periodic sinusoidal fringe pattern in the background (dark frame) that can be substantial compared to other noise mechanisms. An anti-reflective coating can reduce much of this effect, but cannot remove it completely. In addition, all integrated cameras that could be equipped with D-CCD images sensor provide a single tap video output, which severely limits the maximum full frame rate that the unit can generate.

For these reasons, D-CCD technology was also eliminated from further consideration. The detailed photometric analysis concentrated on only sCMOS and InGaAs image sensor technologies.

\subsubsection{Star Radiance}

Star spectral radiance was approximated using a black-body spectral radiance equation from Zombeck [1].

$$
L(\lambda)=\frac{8.48 * 10^{34} * 10^{\left(-0.4 * m_{b}\right)}}{T_{e}{ }^{4} \lambda^{4}\left[e^{\left(1.44 * \frac{10^{8}}{\lambda T_{e}}\right)-1}\right]} \text { Photons } \mathrm{cm}^{-2} \mathrm{~s}^{-1} \mathrm{~A}^{-1}
$$

Where $T_{e}$ is the effective black body temperature of the star. For initial calculations an effective temperature of 5780 ${ }^{\circ} \mathrm{K}$ was used, which relates closely to Main Sequence stars of Class G0 in the Hertzsprung-Russell diagram. Lambda is the wavelength of light in Angstroms. $m_{b}$ is the bolometric magnitude of the star, which is derived from its visible magnitude with a bolometric correction factor to compensate for radiance outside the visible range. Bolometric correction factors were obtained from recent work by Pecaut and Mamajek, 2013 [2]. An even more up to date version is available on line at following site:

(http://www.pas.rochester.edu/ emamajek/EEM_dwarf_UBVIJHK_colors_Teff.txt)

\subsubsection{Sky Background}

GHAPS has requirements to make scientific observations during both night and daytime, therefore the FGS must capture star(s) in both daytime and night for LOS bias offset to be determined. Sky background for nighttime operation is generally not a limiting factor in guide cameras as the typical night sky radiance is lower than visible magnitude 22

GHAPS will operate at an altitude of approximately $30 \mathrm{~km}$, which puts the OTA above a large portion of the atmosphere that scatters daytime sunlight. As such, the daytime sky background irradiance will be significantly lower than the background seen by land-based telescopes. An accurate model of the daytime sky background is needed to properly evaluate the various types of images sensors and their possible windows of operation in the optical spectrum. The MODTRAN6 software simulation package from Spectral Sciences Inc. was used to simulate worst case daytime sky background spectral irradiance levels at $30 \mathrm{~km}$, including the worst sun angle and OTA look angles. The expected daytime spectral radiance shown in Figure 6 is limited to a

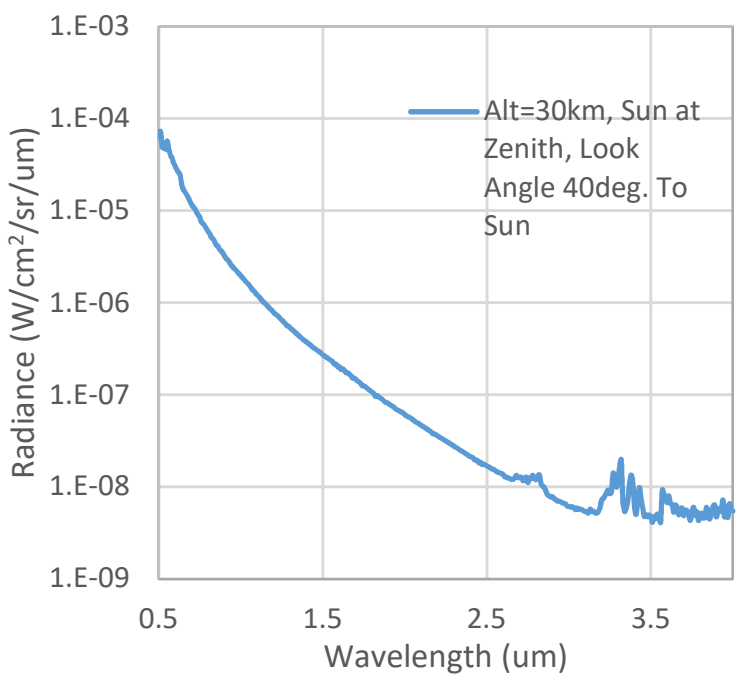

Figure 6. GHAPS Worst Case Daytime Sky Radiance MODTRAN only. 
range from visible to 4 um since sky radiance increases abruptly by 3 to 4 orders of magnitude beyond $4.2 \mathrm{um}$. Good agreement is seen in the plot when compared to similar literature on daytime atmospheric radiance, including the GHAPS Science Instrument Definition Team (SIDT) Report [3].

\subsubsection{Air Glow Emissions}

The MODTRAN simulation package is capable of only modeling radiation transport through the earth's atmosphere using known radiative sources. It cannot predict the simulated emission of electromagnetic radiation that takes place in the earth's upper atmosphere in either daytime or night. Of particular interest is the known bands of emission that occur in the near IR referred to as Airglow. Airglow is caused by various processes in the upper atmosphere such as luminescence by cosmic rays, recombination of solar photoionized atoms, and chemiluminescence from reactions between upper atmospheric molecules. Nighttime airglow spectral radiance is well documented, however little quantitative data on daytime values is available at the altitudes in which GHAPS will be observing.

An estimation of daytime spectral radiance was performed using data obtained from the SABER instrument (Sounding of the Atmosphere using Broadband Emission Radiometry) which is operated aboard the TIMED satellite (Thermosphere Ionosphere Mesosphere Energetics Dynamics). SABER is a radiometric instrument that scans the earth's atmospheric limb, its apparent visual edge, from 0 to over $120 \mathrm{~km}$ multiple times as it orbits. SABER generates radiance measurements at several specific bands in the near to Mid IR (1.25um - $\mathrm{O}_{2}, 1.6 \mathrm{um}-\mathrm{OH}$, and 2.0 - OH) [4]. Based on the literature [5], it appears that Airglow intensity can be assumed to scale linearly across the near IR spectra, i.e. the relative intensity from emission line to emission line remain approximately the same as the overall intensity increases in daytime vs night. With this assumption, the SABER data can be used to obtain a night time to daytime correction factor that can be applied to the well know nighttime Airglow spectra Figure 7 shows a typical plot of the SABER dataset in the bands of interest. Based on an arbitrary seven day average, the worst case daytime to night Airglow ratios appear to be approximately 100 . This estimate is relatively conservative and incorporates the subtraction of daytime MODTRAN sky radiance from the raw SABER data.

Detailed nighttime Airglow spectra was obtained from measurements taken at European Southern Observatory's (ESO) Very Large Telescope (VLT) at Cerro Paranal [6]. ESO provides an online tool to calculate nighttime airglow at look angles from $19.5 \mathrm{deg}$. to zenith. Airglow nighttime spectra with a look angle of $19.5 \mathrm{deg}$. was used, as this is closer to the worst case GHAPS look angles for Airglow. Figure 8 shows the MODTRAN computed daytime sky irradiance with the superimposed daytime Airglow estimate. At certain wavelengths longer than 1 um, the daytime Airglow exceeds the MODTRAN radiance estimates, which will significantly impact the overall performance of near IR image sensors.

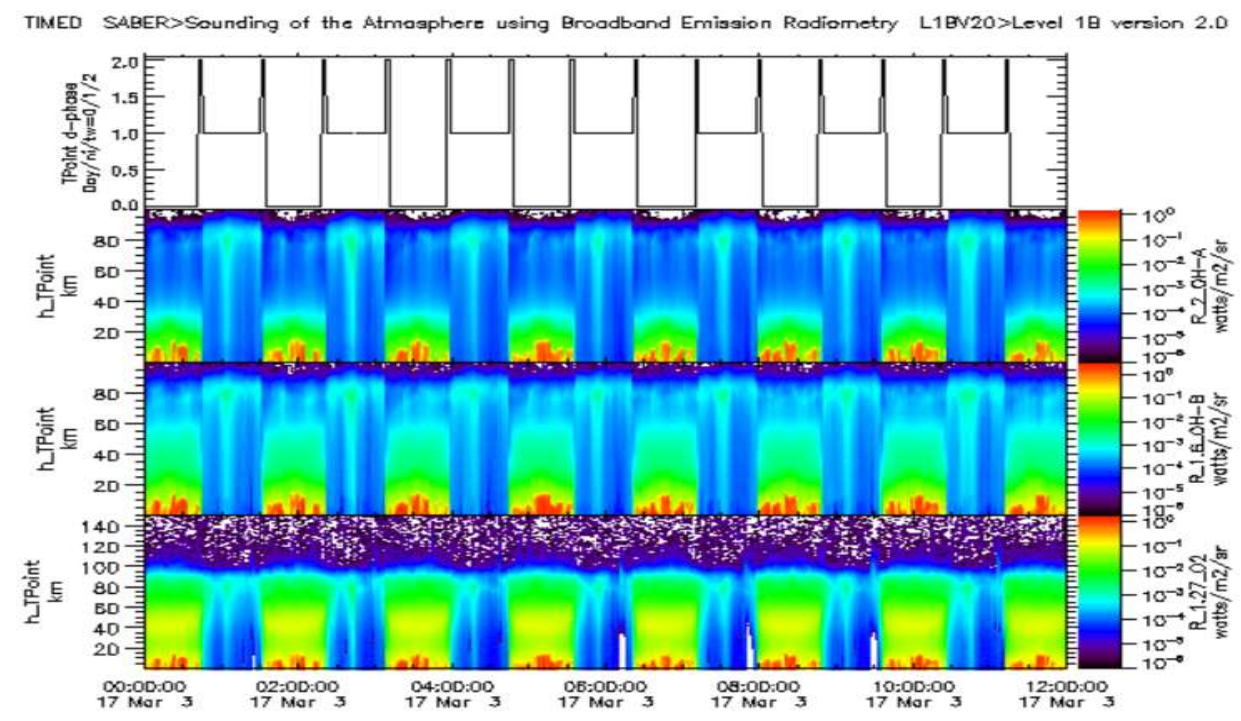

Figure 7. Typical SABER Radiance Data 


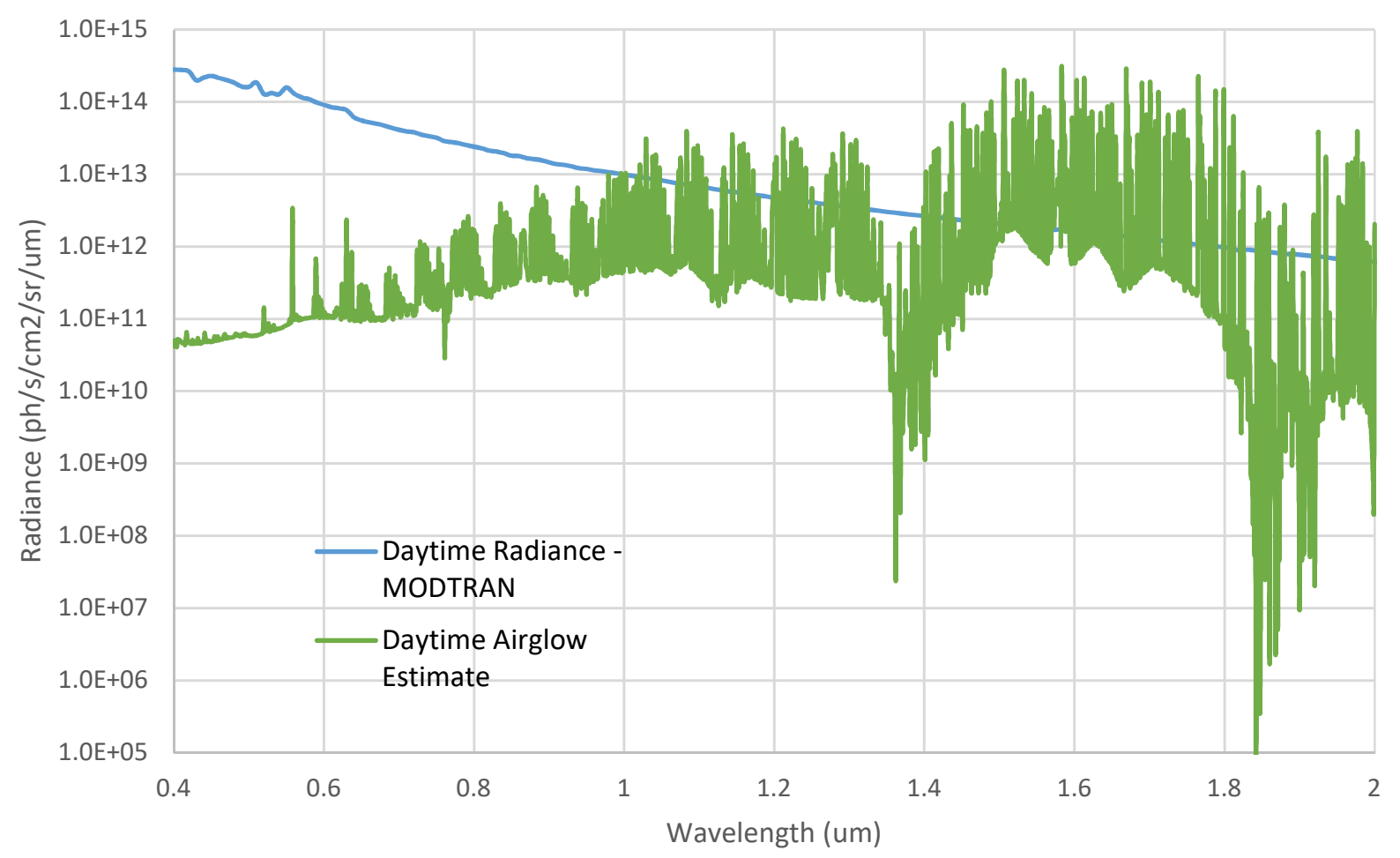

Figure 8. Daytime Sky Radiance Components - 30km, Sun at Zenith, 20 deg. look angle.

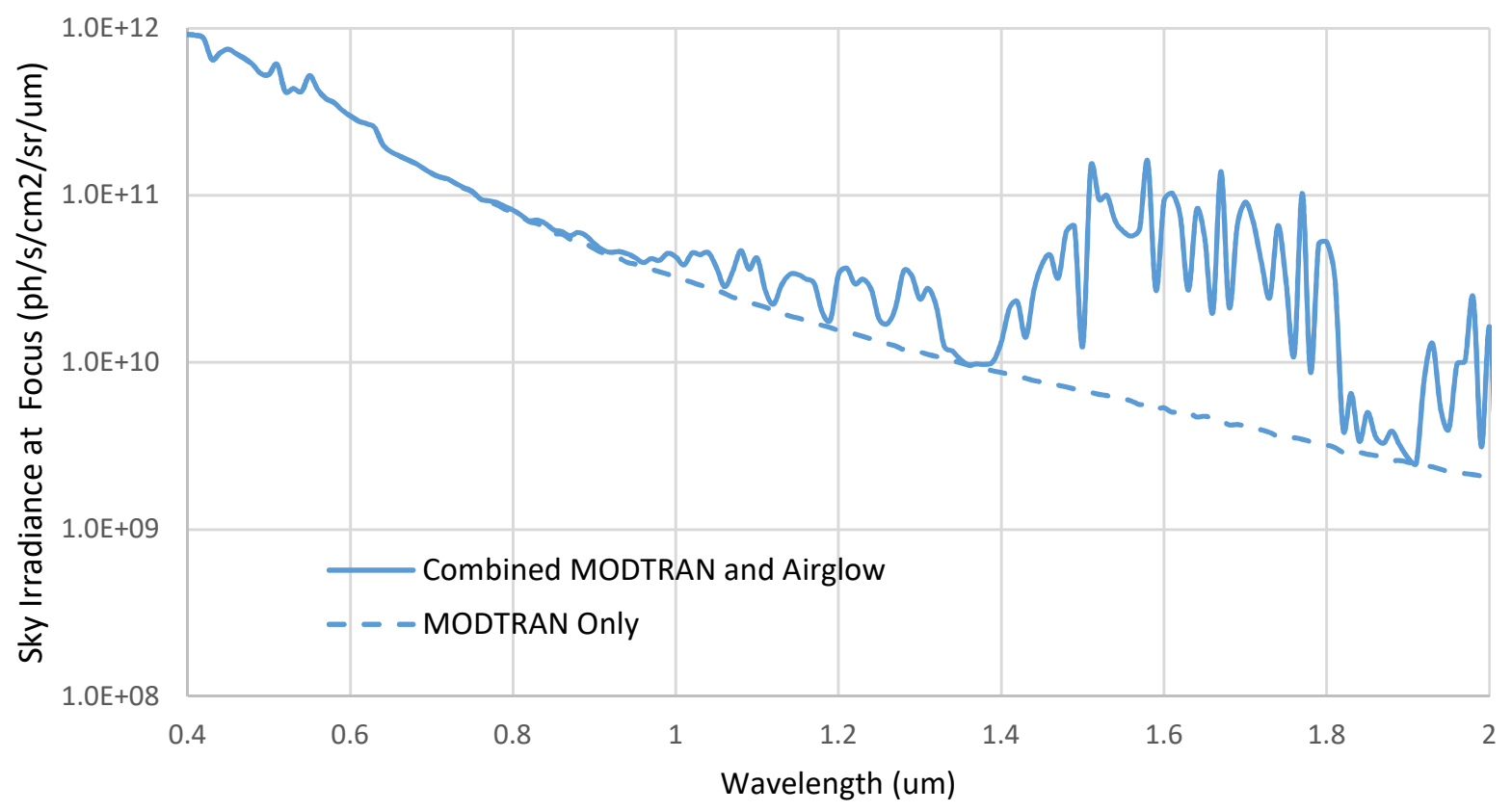

Figure 9. Composite Enveloped Worst Case Sky Irradiance at Cassegrain Focus for GHAPS Photometric Model.

Figure 9 shows the combined MODTRAN and daytime Airglow irradiance at Cassegrain focus in an enveloped form that will be used for calculating each candidate image sensor's performance as a potential device for the FSG. 


\subsubsection{Reimaging Optics}

All image sensors being considered for the FGS have an active area that is smaller than the telescope FOV at Cassegrain focus. The probability for star detection in a random sky FOV is proportional to the limiting magnitude of the FGS and its FOV. To maximize star detection probability, a demagnification of the telescope FOV is needed. Optical models were generated for a two-mirror Korsch and commercially available refractive focal reducers, using design parameters that map the OTA FOV to the full

size of a single FGS candidate image sensor. Results showed that all optical models significantly aberrated the ideal PSF to such and extend that limiting magnitude was made significantly worse. Figure 10 shows a typical PSF for one of the two-mirror Korsch designs.

With these results, the recommendation was to forgo any FGS reimaging optic, and simply place FGS image sensor at telescope Cassegrain focus. FGS optics will consist only of a pick-off mirror and band pass filter. The implication of this design choice is that multiple image sensors and/or cameras may be needed to image enough of the telescope FOV to assure reliable star detection within any random sky section.

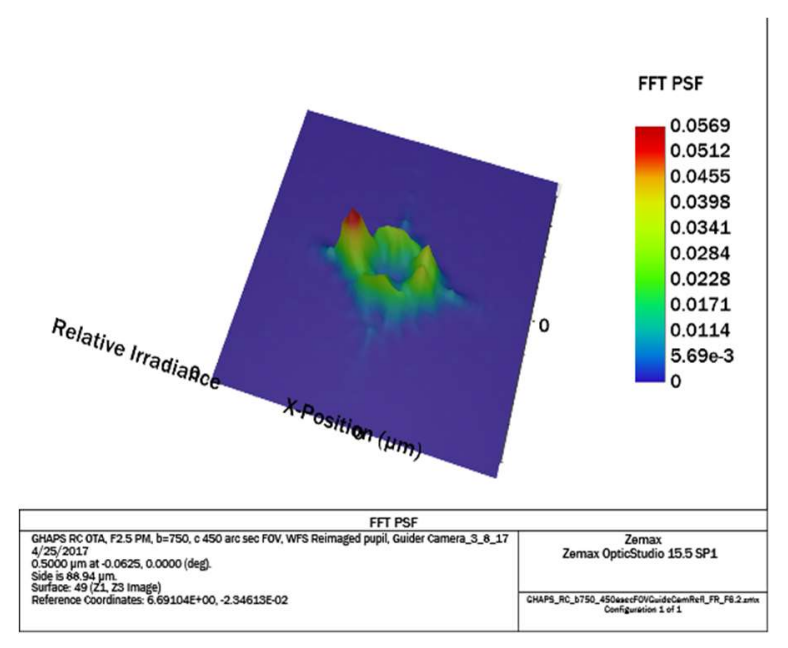

Figure 10. Typical Reimaging Optics PSF Performance.

\subsubsection{OTA Photometry}

For this first order photometry model, the OTA is represented by an idealized 1-meter diameter Ritchey-Chrétien telescope with F/\# ratio of 14.052 , and a central obscuration of $10 \%$ by area. Transforming sky radiance to focal plane irradiance was done using the optical étendue relationship with the form below:

$E_{\text {sky } @ \text { focus }}=\frac{L_{s k y} \pi}{4\left(F \#^{2}\right)} *$ Scope Trans $($ Where Scope Transmission $=0.82)$

Telescope transmission is approximated by multiplying the reflectivity of primary and secondary mirrors with the fractional unobstructed aperture $\left(\mathrm{T}_{\mathrm{scope}}=\mathrm{R}_{\mathrm{M} 1} * \mathrm{R}_{\mathrm{M} 2} * 0.9=0.82\right.$. where $\left.\mathrm{R}_{\mathrm{M} 1}=\mathrm{R}_{\mathrm{M} 2}=0.96\right)$.

Star radiance is transformed into focal plane irradiance by assuming diffraction limited performance, in which all light that is captured by the OTA aperture is concentrated into a single point spread function (PSF) of diffraction limited shape at the focal plane. As is the case for sky background, star radiance is also multiplied by scope transmission. For analysis purposes, a conservative estimate of the imaged star spot size was used. Instead of the usual diffraction limited FWHM, the radius of the first minimum is used, $\mathrm{r}_{\text {Airy disk }}=1.22 \lambda / \mathrm{d}$, where $\mathrm{d}$ is the diameter of the OTA aperture.

In addition to the computations listed above, an adjustable band pass filter was added to the model so that each sensor could be optimized for the best spectral pass band from visible to the mid IR. In all cases, the filter was modeled as an idealized step function at turn on and turn off wavelengths with a $90 \%$ transmission over the band.

\subsubsection{Signal to Noise Ratio (SNR)}

Signal to noise ratio for star images against the sky background will determine the reliable detectability of stars and ultimately the ability of the FGS to determine OTA to Star tracker offset. For the purposes of this analysis, a minimum SNR of 10 has been used. This is a relatively conservative threshold, considering that the WASP pointing system star tracker uses a minimum SNR of approximately 7. The effective SNR was calculated using the following formula: 


$$
S N R_{\text {Star }}=\frac{\text { Signal }}{\text { Noise }}=\frac{Q E * \text { Star }_{\text {ph Airy disk }}}{\sqrt{Q E \text { Star } \text { ph Airy Disk }_{\text {Disel }}+n Q E S k y_{\text {ph } / \text { ixel }}+n \text { Dark }_{\text {noise }} t+n\left(\text { Read }_{\text {Noise }}\right)^{2}}}
$$

Where,

$$
\begin{aligned}
& \text { Star }_{\text {ph Airy disk }}=\text { Total Photons in the Star AryAiry Disk for the specific Integration Time } \\
& \text { Sky } y_{\text {ph } / \text { pixel }}=\text { Sky Backgroun } \frac{\text { Photons }}{\text { pixel }} \text { for the specific Image Sensor and Integration Time } \\
& n=\text { number of pixels occupied by the star airy disc, first minima } \\
& \text { Dark }_{\text {Noise }}=\text { Image Sensor Dark Noise in } \frac{e}{\text { pixel } * \text { sec }} \\
& \text { Read }_{\text {Noise }}=\text { Image Sensor Read Noise in } \frac{e}{p i x e l} \quad(\mathrm{rms}) \\
& Q E=\text { Image Sensor Quantum Effiecincycien } \\
& t=\text { Integration Time }
\end{aligned}
$$

\subsubsection{Sensor Pixel Size}

Pixel sizes for the image sensor being considered ranged from $4.5 \mathrm{um}$ to as large as $20 \mathrm{um}$ square. The size of the image sensor will have a significant impact on the average number of signal electrons/pixel that are generated for a particular image sensor. Given the same Airy disk diameter, a smaller pixel sensor will have fewer photons/pixel as compared to a sensor with larger pixels at the same QE.

Additional consideration must be given to the minimum number of pixels for a star image in order to achieve reliable centroid detection and ultimately a reliable measure of OTA to Star Tracker offset. Based on current star tracker centroiding algorithms, a recommendation of at least nine pixels in area is needed for accurate and reliable star position. Therefore, the photometric analysis assumes at least nine pixels for the area of the Airy disk first minimum, in a format that places at least three pixels across the Airy diameter. The Airy disk is a function of both telescope diameter and wavelength. The midpoint of the band pass filter for the image sensor was used in estimating the Airy disk diameter. Depending on the pixel size and the band pass filter center wavelength, Airy disk areas could be fewer than nine pixels. In these cases, diameters were increased to at least nine pixels so that the centroiding criteria is met and a more realistic side by side comparison is achieved. The implication of a nine pixel Airy disk minimum means that some image sensors with large pixels will require a small amount of defocus to increase the image diameter.

\subsubsection{Sensor Integration Time}

The WASP system will provide a 1 arc sec. pointing accuracy during the nighttime, in which the majority of the error manifests itself as jitter about some average LOS in the cross-boresight plane. At the OTA focal plane, the star image will jitter in a similar fashion, which can smear the star image over a larger area. Sensor integration time has a significant impact on detectable limiting star magnitude. Longer integration times create more star signal electrons per pixel, however they also create larger image smear, which spreads out the star signal over more pixels.

In addition to the FGS determining a bias offset between the OTA and WASP star tracker, the FGS has a desired secondary function to estimate focus quality of the OTA. The OTA WFS system is not a continuous correction system. It requires that GHAPS pause from observing the science object and slew the OTA to a bright guide star for wave front sensing and correction. Knowing when to correct the OTA alignment must come from either a predictive analysis or a measure of image quality during observation. Therefore, if the FGS can provide image quality information in 
addition to its primary functionality, it would provide the means to determine when WFS correction is needed without additional hardware. Bias offset detection relies heavily on centroiding algorithms which are relatively tolerant of image smear, however image quality algorithms are relatively intolerant of image smear.

In a parallel effort, an initial examination of possible image quality algorithms was undertaken. They included direct PSF sampling, autocorrelation, and image sharpness measurements. Autocorrelation was chosen for further evaluation since it is expected to perform well even with a small sampling of the PSF. The accuracy of all these algorithms will be affected by image smear. In order to preserve the potential for the FGS to measure OTA focus quality, some bounds on integration time are needed. Based on anticipated image quality algorithms, a baseline of smaller than $1 / 4$ pixel smear per frame was used. Considering the WASP jitter velocities and minimum pixel size of image sensors candidates is $4.5 \mathrm{um}$, a baseline of $10 \mathrm{~ms}$ integration time has been chosen. This is a conservative estimate, as image sensors with larger pixels could have a proportionally larger integration time and still meet the $1 / 4$ pixel image smear.

\subsubsection{Image Sensor Performance}

Using the assumptions and approximations given in the previous sections for sky background radiance, star radiance, image sensor parameters, and integration time, the daytime focal plane performance was generated for 2 commercially available versions of the sCMOS and InGaAs image sensors. Results are shown in Figure 11. In each case the spectral pass band was optimized for the maximum SNR, and star images were assumed to occupy at least nine pixels in area at the focal plane.

As expected, all image sensors exhibit a slope change when various elements of the SNR dominate the resulting performance. At the brightest star magnitudes, the performance curve flattens due to pixel full well saturation, middle magnitudes are dominated by star shot noise effects, and the dimmest magnitudes are driven by a combination of sky background shot noise and sensor read noise. Using a minimum SNR of 10 as the threshold for FGS tracking algorithms, Figure 11 shows that both sCMOS image sensors give nearly the same performance. The InGaAs image sensors show a marked difference in performance. This can be attributed to the extensive TE cooling and low read noise of InGaAs 2 sensor compared to the InGaAs 1 sensor. (Read Noise of 30 electrons/pixel vs. 170 electrons/pixel)

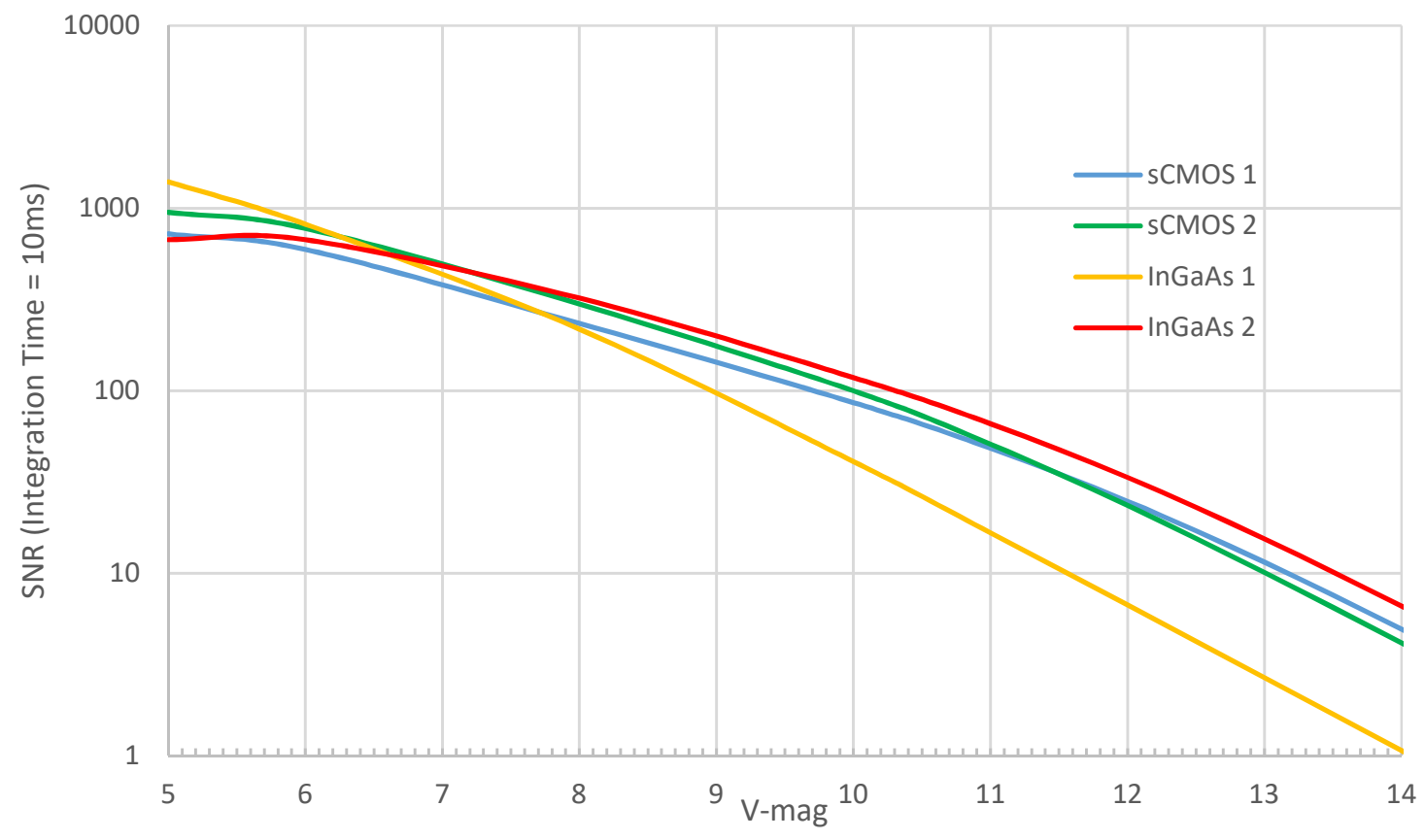

Figure 11. Image Performance Comparison - GHAPS Worst Case Daytime, 10ms Integration Time. 
Using an integration time of $10 \mathrm{~ms}$, both sCMOS and InGaAs will have visible limiting magnitude between 13 and 13.5 for stars that can be used to compute the bias offset. The probability of capturing magnitude 13.5 or brighter stars is dependent on the FOV available to the FGS.

At a SNR of 10 the InGaAs 2 sensor has approximately a 0.3 magnitude advantage over the sCMOS sensors. Using information on mean star density as function of visible magnitude from Zombeck [1] and the maximum FOV available to the FGS, the InGaAs sensor will capture roughly 1.52 stars in a random sky section vs. 1.32 for the best sCMOS sensor, using a $10 \mathrm{~ms}$ integration time. It should be noted that the mean star density listed in Zombeck occur at roughly a galactic latitude of $20 \mathrm{deg}$. Stellar density drops quickly at latitudes above $20 \mathrm{deg}$. to the point where fewer than one star is acquired by the FGS at galactic latitudes higher than $40 \mathrm{deg}$. This leaves a large portion of the sky with a low probability of imaging stars for the FGS, and consequently no bias offset measurement capability.

\begin{tabular}{|c|c|c|c|c|c|c|}
\hline Class & Effective temperature ${ }^{[1][2][3]}$ & Vega-relative chromaticity $\left.{ }^{[4][n b} 1\right]$ & $\begin{array}{c}\text { Main-sequence radius }{ }^{11][8]} \\
\text { (solar radii) }\end{array}$ & $\begin{array}{l}\text { Main-sequence luminosity }{ }^{[1][8]} \\
\text { (bolometric) }\end{array}$ & $\begin{array}{l}\text { Hydrogen } \\
\text { lines }\end{array}$ & $\begin{array}{l}\text { Fraction of all } \\
\text { main-sequence stars }\end{array}$ \\
\hline 0 & $\geq 30,000 \mathrm{~K}$ & blue & $\geq 6.6 R_{\odot}$ & $\geq 30,000 L_{\odot}$ & Weak & $\sim 0.00003 \%$ \\
\hline B & $10,000-30,000 \mathrm{~K}$ & blue white & $1.8-6.6 R_{\odot}$ & $25-30,000 L_{\odot}$ & Medium & $0.13 \%$ \\
\hline A & $7,500-10,000 \mathrm{~K}$ & white & $1.4-1.8 R_{\odot}$ & $5-25 L_{0}$ & Strong & $0.6 \%$ \\
\hline $\mathbf{F}$ & $6,000-7,500 \mathrm{~K}$ & yellow white & $1.15-1.4 R_{\odot}$ & $1.5-5 L_{\odot}$ & Medium & $3 \%$ \\
\hline G & $5,200-6,000 \mathrm{~K}$ & yellow & $0.96-1.15 R_{\odot}$ & $0.6-1.5 L_{\odot}$ & Weak & $7.6 \%$ \\
\hline К & $3,700-5,200 \mathrm{~K}$ & light orange & $0.7-0.96 R_{\odot}$ & $0.08-0.6 L_{\odot}$ & Very weak & $12.1 \%$ \\
\hline M & $2,400-3,700 \mathrm{~K}$ & orange red & $\leq 0.7 R_{\odot}$ & $\leq 0.08 L_{\odot}$ & Very weak & $76.45 \%$ \\
\hline
\end{tabular}

Figure 12. Harvard Stellar Class Distribution of Local Milky Way Galaxy Stars [8]

\subsubsection{Potential InGaAs Advantage with Star Spectral Radiance}

The previous photometric analysis was conducted assuming all stars have the same spectral radiance content, class G0 stars with effective temperature of $5780 \mathrm{deg}$. K. The actual sky will have stars with a wide range of effective temperatures. The predicted distribution of effective star temperatures within our galaxy has been well documented $[7,8]$, and is shown in Figure 12. The majority of stars appear to have relatively low effective temperatures, which places a higher portion of their spectral radiance in the near-IR band. Considering the spectral sensitivity of InGaAs and sCMOS sensors, the possibility exists that InGaAs may have a significantly better detection capability for lower effective temperature stars than sCMOS, ultimately leading to a significantly higher probability for star detection over more of the sky.

The potential spectral advantage of InGaAs was investigated by first utilizing the same photometry model used to determine SNR vs. Visible Magnitude. By varying the range of effective star temperatures in the model and determining the stellar magnitude when SNR equals 10, a curve of each sensor's limiting magnitude ( $\mathrm{SNR}=10)$ vs. effective star temperature was generated. Figure 13 show the results for sCMOS and InGaAs. Figure 14 show the difference in limiting magnitude performance between InGaAs and sCMOS. The performance difference may seem relatively small, but if the majority of stars have effective temperatures lower than $3800 \mathrm{deg}$. K, then InGaAs has an advantage of over one magnitude compared to sCMOS. This equates to a doubling of the capturable star density.

Rather than using a predictive estimate of star spectral class distribution, analysis went forward using actual star catalogs that have the capability to reach at least magnitude 17 stars. Several catalogs were considered, but the NOMAD star catalog was chosen due to its combination of star quantity and spectral magnitude at both visible and near IR photometric bands. The NOMAD catalog is a combination of Hippacros, Tycho-2, UCAC-2, USNO-B1, and 2MASS surveys, and contains over 1 billion star down to visible magnitude 22.

Obtaining and working with the entire NOMAD catalog is difficult and time consuming due to its size. As a compromise, a sub-sample of the NOMAD catalog was used. Using the NOMAD online search capability, cone search data for many 1 degree radius fields were obtained. Cone searches were obtained for each galactic latitude in 10 deg. increments and 4 searches (longitude 0,90. 180, and 270) at each galactic latitude. This yielded star sample sizes at each latitude from 16,000 at the poles to more than 180,000 near the equator. 
Figure 15 and Figure 16 show relevant statics for the NOMAD star subset collected at a galactic latitude of 10 deg. Although the plot of star count vs visible magnitude exhibits the constant log slope as expected, the plot of stellar spectral class count differs significantly from that expected by literature. In both the NOMAD and its subset 2MASS, the fraction of cooler $\mathrm{K}$ and $\mathrm{M}$ class stars is much lower than expected. This is also consistent with findings from another catalog that was initially considered called APASS. This discrepancy was never fully explained, but it appears that the cataloging of cooler stars may be limited by the inherently lower visible and near IR magnitudes that they possess. As a result, InGaAs sensor will likely not have the significant advantage over sCMOS as first hoped. Nonetheless, a quantitative performance analysis of the two sensors was still carried out using the NOMAD catalog.

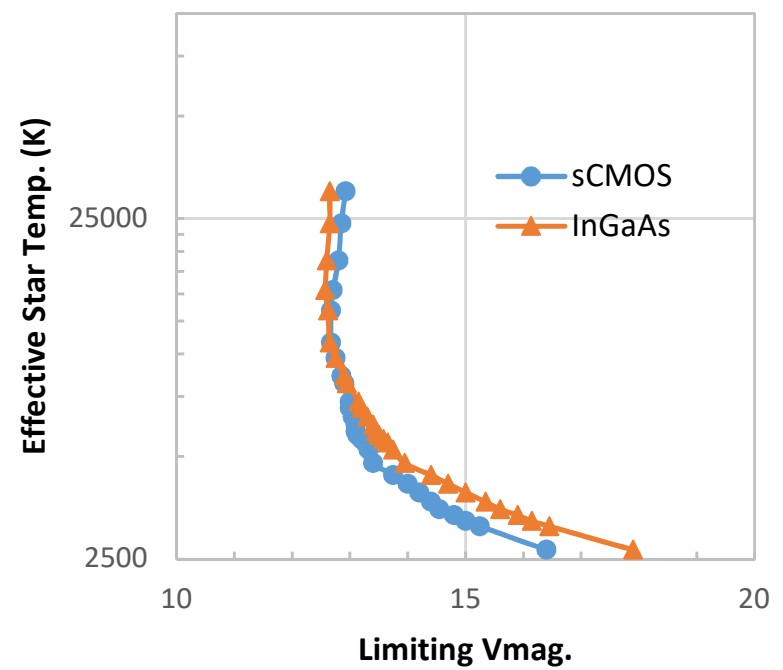

Figure 13. InGaAs and sCMOS Limiting Magnitude $(\mathrm{SNR}>10)$ as a function of Star Effective Temperature.

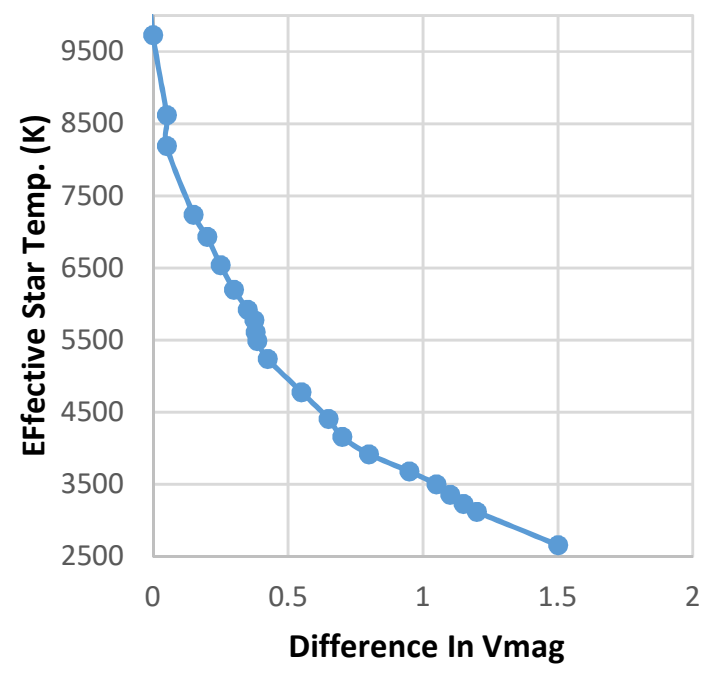

Figure 14. Limiting Magnitude (SNR>10) Advantage of InGaAs over SCMOS

A relationship between photometric bands V, J, and effective star temperature were obtained using data from Pecaut and Mamajek [3]. For each star, the V-J value was obtained and translated into an effective temperature. The FGS limiting magnitude was generated for each catalog star from the V-J relationship and the curve fit described in section 3.1.10. Star visible magnitude is then compared to FGS threshold using the star effective temperature. If the star visible magnitude is smaller (brighter) than the FGS threshold, the star can be imaged with a SNR $>10$. This was performed for both sCMOS and InGaAs sensors. Predicted FGS star densities at periodic galactic latitudes were then

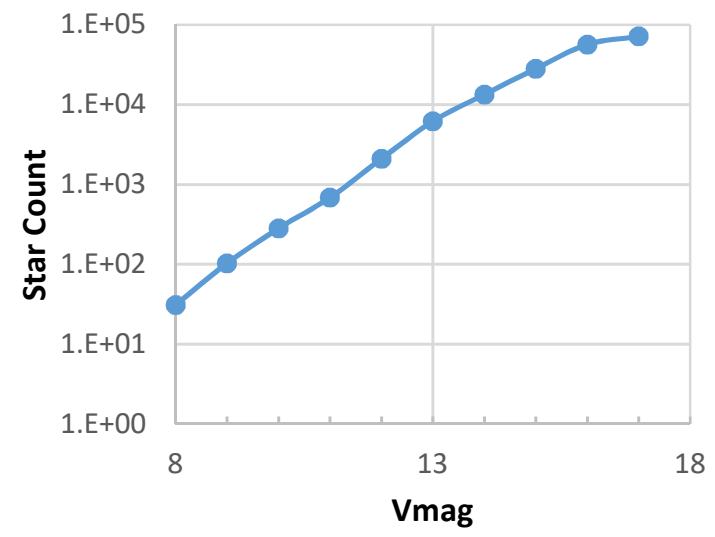

Figure 15. NOMAD Catalog Subset Star Count vs. Visible Magnitude.

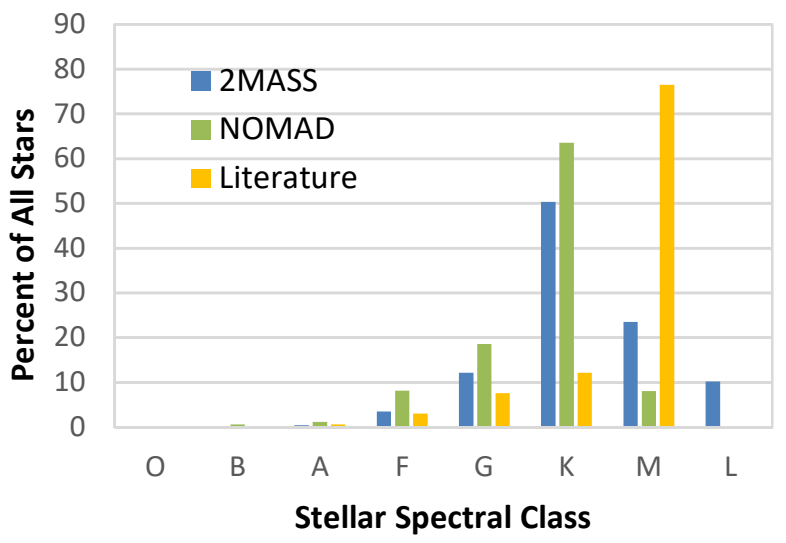

Figure 16. Stellar Spectral Class Distribution. 
generated by tallying the stars with visible magnitudes brighter than the limiting magnitude of each sensor.

Results are shown Figure 17. True star effective temperature can be affected by three main factors. Star surface gravity, star metallicity, and reddening from interstellar dust. Surface Gravity and metallicity require detailed spectral scans of the star in question to quantify, so it cannot be applied in this analysis. In lieu of this potential error, these factors were estimated, using information from M. L. Houdashelt, R. A. Bell, 2000 [9]. The maximum errors were found to be $\pm 150 \mathrm{deg}$. $\mathrm{K}$ in Effective Temperature, which relates to approximately $\pm 8 \%$ in calculated stellar densities. This level of uncertainty should not significantly affect the results shown in Figure 17.

Results show that InGaAs may have substantial performance gains at lower galactic latitudes, but at latitudes above 50 deg. the advantage is negligible. Based on Figure 17, InGaAs would be the best choice, however other factors such as size, weight, power, and cost are actually in the favor of sCMOS. Recall that the FGS will not have any reimaging optics to map the maximum telescope FOV to the image sensor. Instead the image sensor must cover the largest portion possible of the FOV available to it at Cassegrain focus. To do so with InGaAs sensor will require at least three image sensors and cameras. The same may be possible with fewer sCMOS sensors due to their larger array size. The desired functionality of focus quality measurement is also best served by sCMOS arrays vs. InGaAs due to their smaller pixel sizes compared to InGaAs arrays.

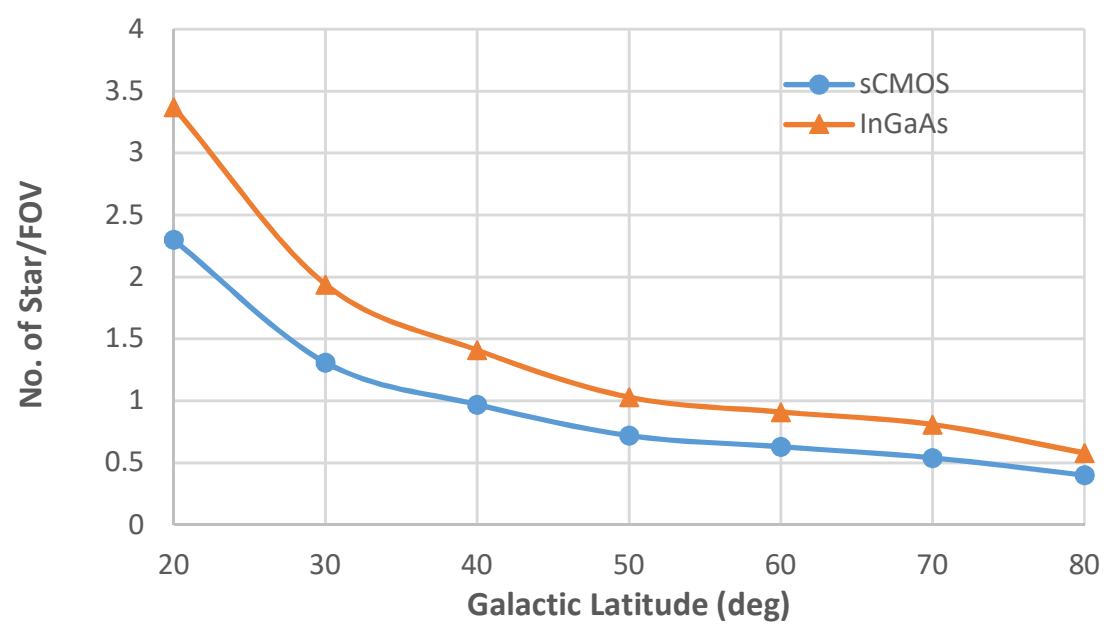

Figure 17. Predicted FGS Star Count for InGaAs and sCMOS - 10ms Integration Time, Maximum FGS FOV, Optimized Spectral Passband.

The size and power consumption of InGaAs is 2 to 3 times that of the sCMOS solution, plus the cost of InGaAs is 3 to 4 times that of sCMOS. For these reasons, sCMOS was chosen as the image sensor technology to implement as an FGS.

\subsubsection{1 sCMOS Camera Selection}

With sCMOS chosen as the best option for the FGS, work began to determine the best sensor and camera(s) for a detailed design implementation. An industry survey was conducted for the best sCMOS camera candidates, which weighed the advantages and disadvantages of many cameras factors. The factors included performance, power, size, weight, cost, etc. Candidate cameras included models from Hamamatsu, Andor, Raptor Photonics, Nikon, Basler, Ximea, Finger Lakes Inc., QHYCCD, ZWO, and SBIG. After weighing the advantages and disadvantages of each candidate, only 3 units warranted continued development with the FGS. The best sCMOS camera candidates included the following: 
1) Finger Lakes Inc. - KL400

2) Ximea - CB152MS-GP-X4G2

3) Andor - Zyla 5.5 (Improved QE, 80\%)

Without any reimaging optics, the FGS must capture as much of the available FOV as possible, which requires multiple cameras in all cases. Figure 18 shows an overlay of the OTA FOV with the best 4 options of FGS sensor layout using the sCMOS cameras listed above.

\subsubsection{Extended Integration Time}

The results in Figure 17 show that even when imaging the maximum available FOV for the FGS, using a 10ms integration time, neither image sensor technology will be capable of delivering reliable star imaging over the full sky. This leads to a different approach in which significantly longer sensor integration time is needed. As stated earlier, longer integration times allow more star signal photons to accumulate in the pixels contained in the PSF, but that additional time allows jitter to spread out the PSF, which offsets the benefit. To estimate the star image smearing effect, information is need about the WASP cross-boresight jitter as a function of time. As part the GHAPS project, a medium fidelity simulation model of the WASP and OTA has been developed. Using simulation data obtained from a typical target angle over a 60 second period, high resolution pointing jitter data was derived. The results were statistically characterized to obtain jitter velocities that were included into the photometry model. Jitter was characterized as 1 sigma and 3 sigma values. One sigma jitter velocity values were used to compensate the photometric model, which is a reasonable value that encompasses $>90 \%$ of expected jitter during trackin

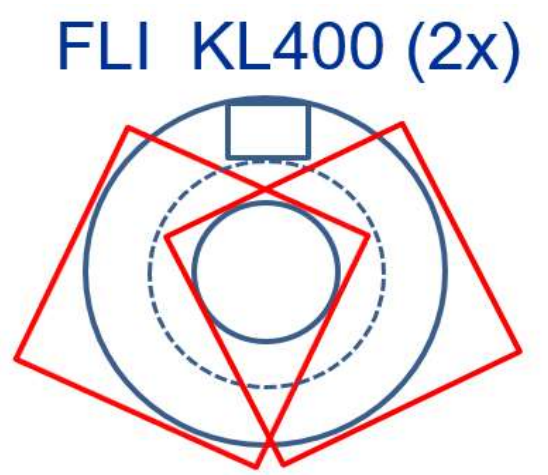

8.7 e4 arc sec 2

Ximea $(3 \mathrm{x})$

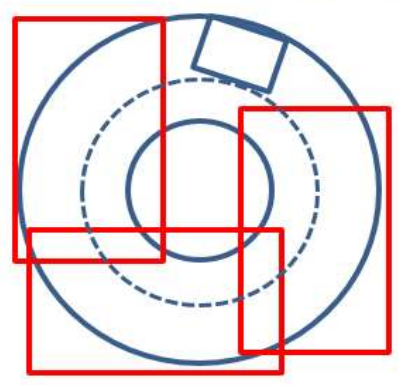

$9.1 \mathrm{e} 4$ arc $\mathrm{sec}^{2}$

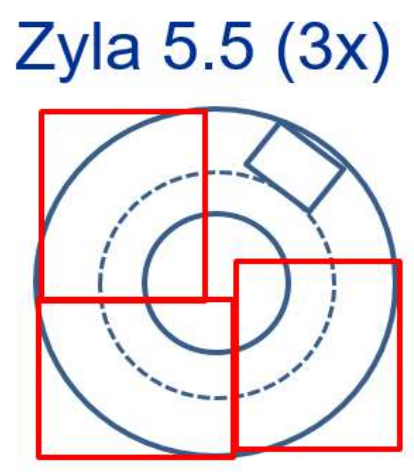

$7.8 \mathrm{e} 4 \operatorname{arc~sec}^{2}$
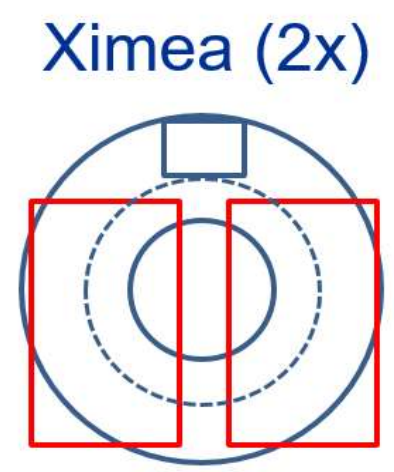

6.5 e 4 arc sec ${ }^{2}$

Figure 18. Image Sensor Layouts for FGS FOV Coverage 


\subsubsection{Photometric Analysis Conclusion}

Results in Figure 19 show that at least two of the configurations (2x FLI and 3x Ximea) are capable of capturing at least 1 star at galactic latitudes up to $80 \mathrm{deg}$., which is greater than $98 \%$ of the sky. Additionally those same camera configurations would also yield greater than 2 stars at galactic latitudes higher than 55 deg., which is more than $80 \%$ of the sky. From a mass and power consumption standpoint the FLI configuration is best, however the use of non-orthogonal angles in the two-camera orientation could become difficult to transform and merge if the FGS must operate at relatively fast frame rates. Current expectations for FGS measurement period is on the order of $60 \mathrm{sec}$., which should be adequate to accommodate the extra computation which the FLI configuration may need. Hence, the two-camera FLI configuration is recommended for FGS implementation, if this functionality is to be part of the GHAPS facility.

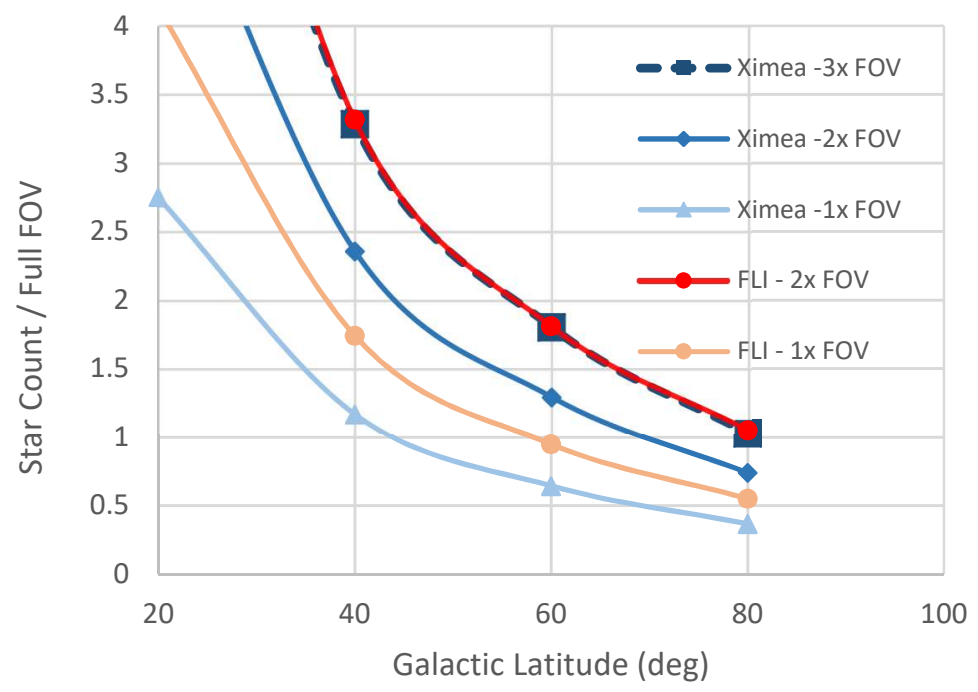

Figure 19. Predicted FGS Star Count Performance - 2 sec. Integration Time, FOV as shown in Figure 18, 1 sigma Jitter Velocities.

\subsection{Pointing Requirements}

In light of the potential pointing bias offset issue, a re-examination of the GHAPS pointing requirements was undertaken in parallel with the FGS trade study. The results yielded modifications to existing requirements and additional new pointing requirements for GHAPS. Some of the key changes included the following:

1) A separation of pointing requirements into short term (jitter) and longer term (bias).

2) WASP and OTA will provide an absolute point error of less than 45 arc sec. $(3 \sigma)$.

3) Given a target relative offset (bias offset), GHAPS shall point the mean optical axis of the telescope within 0.1 arc sec. $(3 \sigma)$ of commanded position.

Item 2 above indicates the maximum possible bias offset that could be present at Cassegrain focus due to deflections and deformations in the OTA. Item 3 above reflects the long term pointing accuracy needed to meet mission success. Item 3 was derived by a detailed examination of potential science missions listed in the GHAPS SIDT Report. Therefore, any FGS system will need to measure bias offset to an accuracy of better than 0.1 arc sec.

\subsubsection{FGS Measurement Accuracy}

An estimate of the FGS measurement accuracy was undertaken, based on the configuration recommended in Section 0 . In order to estimate the measurement accuracy, some notional algorithm is needed for computation. The FGS camera will need to operate with multiple integration times to avoid saturation effects. Two integration times can be assumed, one optimized for the dimmest stars (approximately $2 \mathrm{sec}$.), and one for bright stars (approximately $1 \mathrm{msec}$.). These images will be combined after acquisition, and adjusted to create a single very high bit depth image with all imageable stars 
available for FGS algorithm processing. The FGS will utilize a simple center of mass centroid algorithm to estimate star position within the image. The centroiding algorithm accuracy will be susceptible to image jitter. Based on the estimated WASP jitter frequencies and the FGS camera integration time, errors could be significant enough to warrant additional time averaging in software beyond $2 \mathrm{sec}$. to improve accuracy. In this case, the FGS will need to perform additional processing to effectively co-add individual frames to create an extended duration image that can be used for a Lost-InSpace pattern matching algorithm which determines the FGS FOV in celestial coordinates. Using the WASP jitter simulation as a function of time and a basic model of the star PSF, synthetic time-lapse images of the stars were generated for various integration time from 1 to $60 \mathrm{sec}$. Some of the time lapse images are shown in Figure 20.

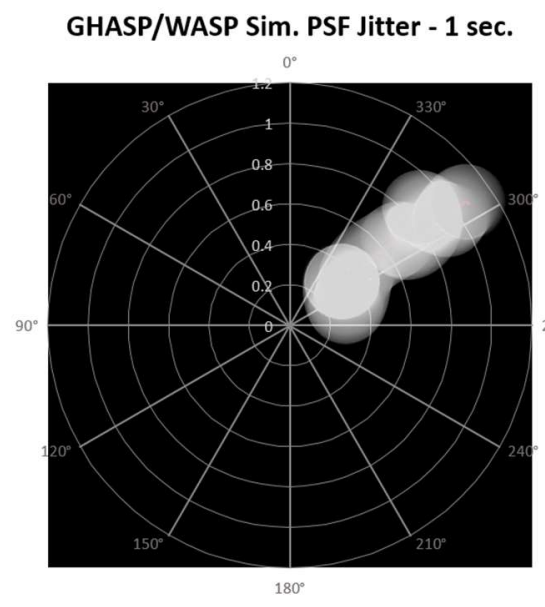

GHAPS/WASP Sim. PSF Jitter - 10 sec.

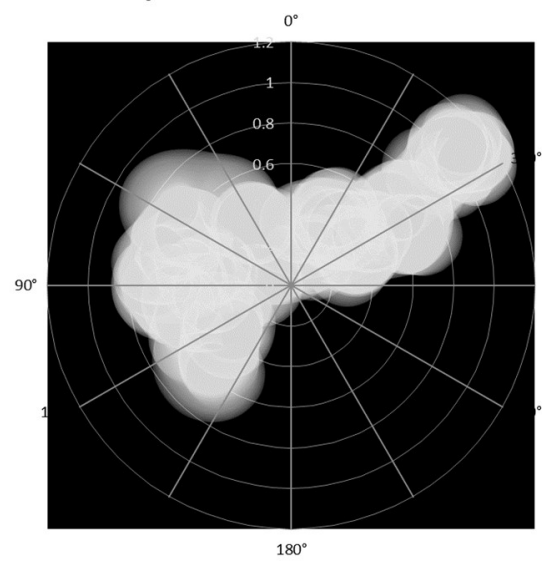

GHAPS/WASP Sim. PSF Jitter - 5 sec.

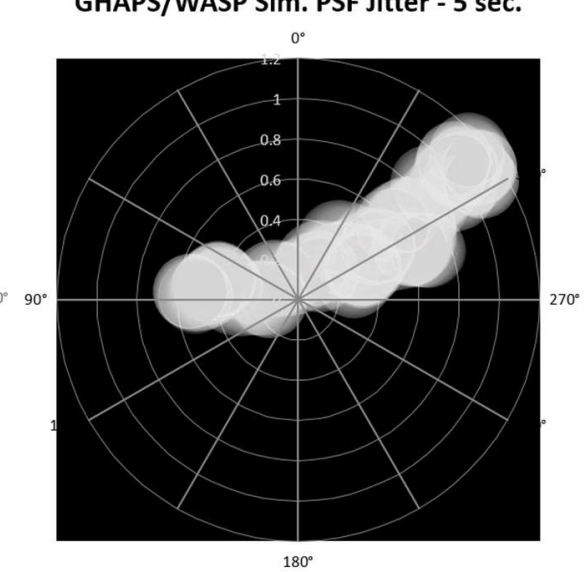

GHAPS/WASP Sim. PSF Jitter - 60 sec

$0^{\circ}$

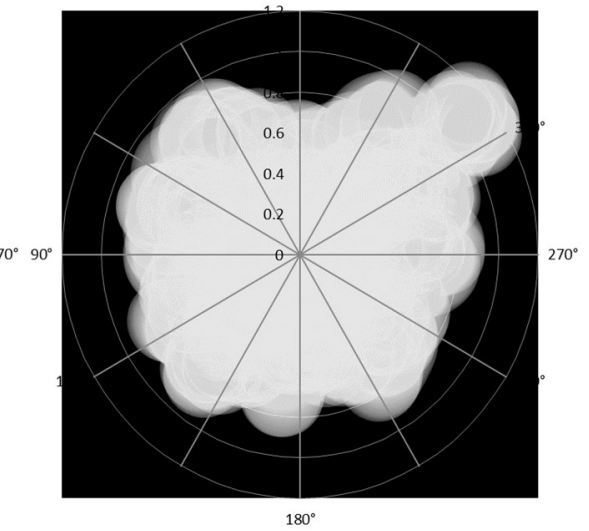

Figure 20. Synthetic PSF Image Smear for GHAPS/WASP Pointing Jitter Simulation (Magnitude Axis is in arc sec.)

Using a simple center mass weighted centroiding algorithm, the computed star position was then compared to the cross boresight origin. The results are shown in Figure 21 as the noise equivalent error angle (NEA) for the FGS in arc sec. NEA values were calculated using the process outlined in Liebe, 2001 [10]. NEA values were computed for two camera configurations with a single star capture (FL400 $=11 \mathrm{um}$ pixel, Ximea $=4.5 \mathrm{um}$ pixel), which yields the worst case NEA. Additional star capture improves NEA, and a 3-star case is shown for reference. NEA does not represent all sources of error that could contribute to FGS accuracy, but it is the largest inherent error source that cannot be calibrated out or reduced by additional processing. In all cases the FGS system appears to be capable of delivering better than $0.1 \mathrm{arc} \mathrm{sec}$. using accumulated measurement time greater than approximately $10 \mathrm{sec}$. 


\subsubsection{FGS Alignment Errors}

The NEA shown in Figure 21 assumes that the FGS optical components are ideally mounted to the OTA with no misalignment occurring during flight. Gravity and thermal effects are expected to cause deflections in flight at the mounting points for FGS components, however a quantitative analysis that included the OTA and Instrument Deck as unavailable at the time of this trade study. As a means to understand the FGS system misalignment, a notional FGS optical component layout was consid-

ered where the FGS pickoff mirror is located along the OTA optical axis at a 45-degree angle. (refer to Figure 22) The FGS Camera is located approximately $50 \mathrm{~mm}$ from the pick-off mirrors centerline. Any FGS band pass filters are built into the camera housing.

Assuming rigid bodies for the pick-off mirror and Camera, the angular and displacement sensitivities were calculated for each component. The pick-off mirror was found to have an angular sensitivity of 3.6 marc sec./arc sec. and displacement sensitivity of 0.01 arc sec./um. The camera will have a displacement sensitivity of $0.015 \mathrm{arc} \mathrm{sec} . / \mathrm{um}$.

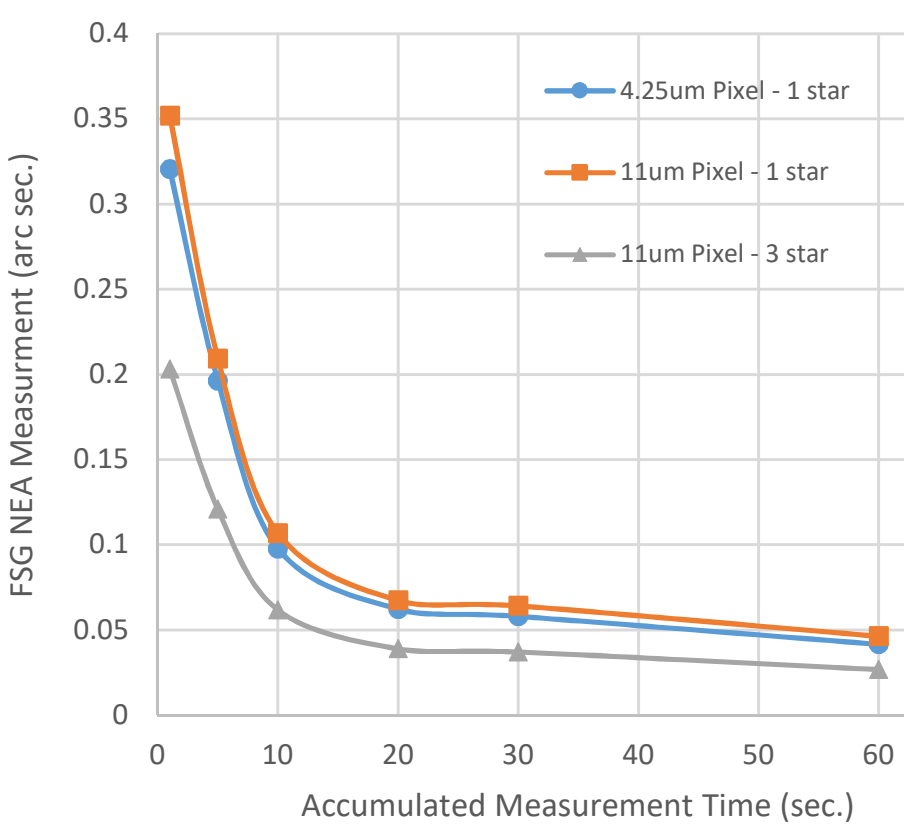

Figure 21. Estimated FGS Measurement NEA for sCMOS Configurations.

The misalignment sensitivities are high in some cases. As an example, if a camera displacement of 10 um occurs during tracking, as much as 0.15 arc sec. of misalignment is seen in the FGS FOV, which is beyond the required FGS system performance. The in-flight misalignment errors of the FGS optical components cannot be corrected and will contribute directly to the directly to the overall system error.

\subsection{FGS Implementation}

The previous photometric analysis assumed that the GHAPS OTA and Avionics would provide the hardware and software needed for the FGS. As an alternate approach, the possibility of implementing the bias offset measurement capability in the Science Instrument was considered. The GHAPS Science Instrument (SI) is a large high-performance instrument package $(200 \mathrm{~kg}, 1$ cubic meter) that provides the capability to accomplish the GHAPS science mission objectives. The SI developers have the freedom to design a single or multi-instrument package that best meets the science mission objectives. The SI could be modified or completely swapped out for another SI should the project require it for future missions.

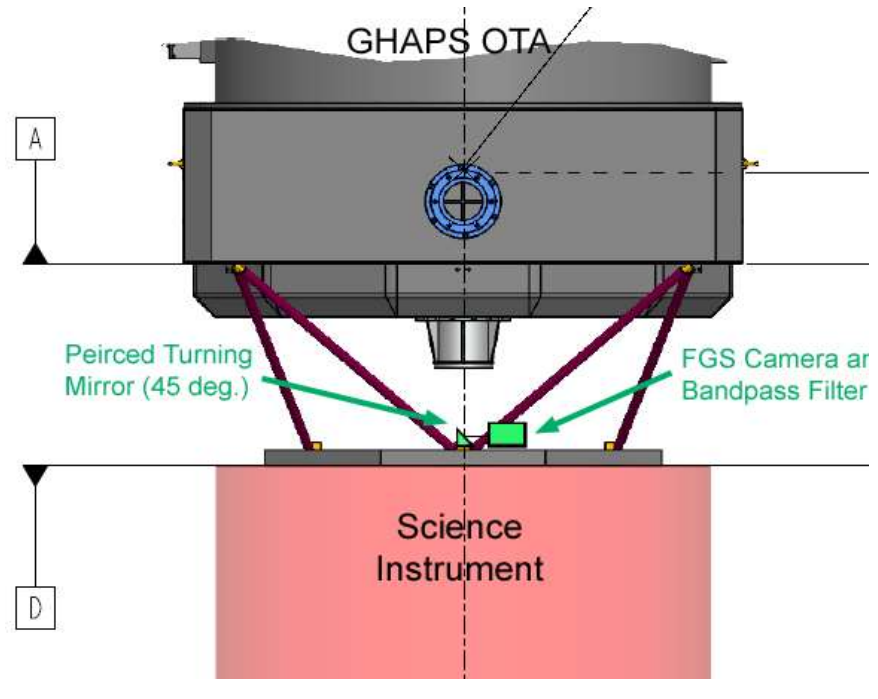

Figure 22. Notional FGS Optics Configuration (approximate scale for FGS components) 
Using the SIDT report as a guide for possible science missions, the most likely form that a GHAPS SI will take is either an imaging instrument (focal plane array) or a non-imaging instrument, such as a spectrometer. Imaging instruments will inherently have star imaging capability similar to that needed for the FGS, depending on its FOV. Similarly, almost all conceivable spectrometer-type instruments will also need imaging capability to facilitate continuous spectrometer slit placement. The possibility exists that SI designers could incorporate pointing bias offset measurement capability within the SI without major impacts to the basic SI design.

\subsubsection{SI vs. FGS Performance}

The key performance measures for bias measurement are detectable stars per FOV and NEA. These factors have been estimated for the recommended FGS configuration and found to be adequate using commercial scientific imagers. Assuming the same hardware components and processing algorithms are available to the SI designers, similar performance should be attainable. For detectable stars per FOV, SI-based systems should have an advantage compared to the FGS. The FGS optical configuration places the pickoff mirror at least $50 \mathrm{~mm}$ before Cassegrain focus. At this point in the optical train, the ray bundle for a point source has an approximate diameter of $44 \mathrm{arc} \mathrm{sec}$. The OTA Science FOV (180 arc sec. diameter) must be preserved without any artifacts or vignetting from the FGS pick off mirror. To avoid vignetting any star ray bundle, the pickoff mirror must not encroach on the vignetting buffer zone that exists around the Science FOV, as shown in Figure 23. This limits the available FOV for the FGS to roughly $9.0 \times 10^{4} \mathrm{sq}$. arc sec. In contrast, an SI-based system can be located closer to the focal plane and have

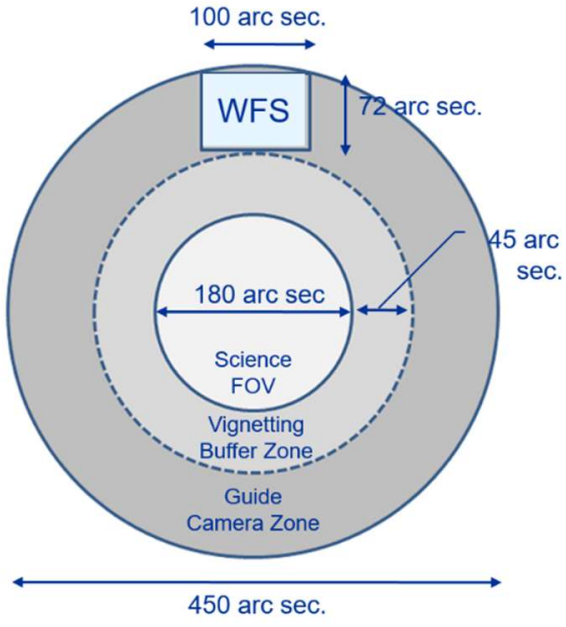

Figure 23. GHAPS OTA FOVs at Cassegrain Focus fewer restrictions on vignetting keep out zones. In the case of a high-performance SI focal plane array, the same array has the potential to be used for bias offset measurement, in which case the entire FOV outside the SI is available.

NEA is a function of FOV, star centroid error, and the number stars detected. Assuming an SI-based system could have the same components, NEA should be equal to the FGS system or potentially higher since the available FOV could be larger.

The uncorrectable errors that affect the FGS optical components, will still be a factor for SI-based systems, however due to the tighter mechanical and thermal coupling of the same components to the desired SI FOV, in-flight misalignment impacts are expected to be reduced. SI-based guider optical components will be located much closer to the SI internal focal planes, such as the SI Science image sensor or Science spectrometer slit, hence misalignment sensitivities are lower. In addition, SI-based guider optical components will likely be mounted to the same optical bench as the SI detectors.

\subsubsection{SI vs FGS Size Weight and Power (SWAP)}

The current volume allotted for the SI should be adequate to accommodate an SI-based system. Based on similar balloon payload instruments on the Balloon Observation Platform for Planetary Science (BOPPS) [11], which did employ a separate guide camera, the additional volume is well within the allotted volume for the GHAPS SI. Therefore, neither FGS nor SI-based systems should impact the current GHAPS volume constraints.

The main difference in mass between SI-based and FGS systems lies in the required cooling lines. The current GHAPS design allows for up to $400 \mathrm{~W}$ of cooling capacity for the SI, which includes cooling lines from the GHAPS gondola to 
the SI. An FGS system will required additional cooling lines to be run from the gondola or SI to the FGS cameras, that will increase overall mass of this configuration.

Power consumption between the 2 options is considered to be equal.

\subsubsection{SI vs FGS Cost and Schedule}

The current FGS concept is driven by satisfying all possible science missions so as to achieving the best possible performance with the least available FOV for guiding. SI developers are expected to optimize their designs to a tighter range of science targets for each observation campaign. This allows SI designers to optimize their SI guider designs in the same way, which could lead to a less costly configuration compared to the FGS. This design flexibility combined with a larger FOV available for guiding gives the SI-based systems a slight cost advantage.

Requiring the SI to provide its own bias offset measurement capability is not trivial and will impact resources of any contractor. Much of the SI-based guider tasks can be performed in parallel with the SI main instrument development, but regardless the contractor schedule will be impacted. The level of impact is very dependent on the contractor's workforce size and/or experience base.

\subsubsection{SI vs FGS Mission Operations}

To assess the potential impact on system operations a variety of modes were considered that range from fully automated to others that have some level of manual involvement. The most likely modes were narrowed to FGS automated, SI automated, and a semi-automatic mode. Automated FGS is considered the baseline mode, and assumes that GHAPS will acquire process FGS images to determine the OTA's center of FOV using a limited Lost-in-Space (LIS) algorithm that determines celestial coordinates by matching images to a detailed star catalog. An offset is then provide to the WASP to correct bias error. The automated SI mode is very similar in operation, but the SI will acquire the images, potentially process them, and correct bias offset using an internal fine steering mirror. The semi-automatic mode version emphasizes a concept where minimal SI processing is needed and unburdens the SI from some image processing and all of the LIS computation. A single or set of high bit depth images are downlinked to the ground, additional image processing and the LIS calculation is carried out on the ground. The offset is uplinked to the WASP and corrected.

The Semi-Automatic process lends itself to situations where the camera captures only a single star in the FOV. Automated LIS processing requires at least 2 stars in the FOV. When only 1 star is visible, some manual intervention is needed to determine its identity and celestial coordinates before it can be used for bias offset measurements during tracking observations. Even the FGS will require manual intervention if FOV star count is fewer than two stars, therefore the SIbased and FGS type systems are essentially equal in terms of negative impact on GHAPS operations concepts.

\section{IMPLEMENTATION RECOMMENDATION}

There is understandably a desire to divorce the bias detection capability from the SI, so that the GHAPS system is completely self-sufficient in terms of pointing correction. It simplifies the interface to the SI and does provide the capability for GHAPS to perform WFS correction without SI involvement. However, with exception of schedule impact, an SI-based guider is expected to have equal or better characteristics for all factors being considered. Of particular concern is the uncorrectable errors that come about from guide system optical component deflection. Using the misalignment sensitivities estimated for the FGS, even a very small deflection exceeds the measurement accuracy requirement. The SIbased systems will inherently have lower sensitivities to deflection, and is the best implementation from an engineering and performance standpoint. In general, having the full design trade space in the hands of the SI developer makes for an 
optimized bias measurement system that is not over designed or underperforming. The main drawback to a SI-based system is that it burdens the SI developer with additional work and cost. Any additional future SI for GHAPS must also provide the same form of bias offset measurement, ultimately impacting long term program costs.

Most of the candidate science missions discussed in the SIDT Report will require active pointing correction for jitter performance better than WASP can deliver. The majority of jitter compensation solutions will require some form of guiderlike imaging capability within the SI. SI designers should be able to augment these systems to perform the bias correction as well. This configuration tends to be the most prevalent in similar class observatory systems. For these reasons an SIbased bias offset measurement system is recommended for GHAPS implementation, and the project should allow SI developers options in the level of automation for system operations.

\section{References}

1. Zombeck, M., "Handbook of Space Astronomy and Astrophysics, $2^{\text {nd }}$ Edition", Cambidge University Press, 1982

2. Pecaut, Mark J.; Mamajek, Eric E., "Intrinsic Colors, Temperatures, and Bolometric Corrections of Pre-main-sequence Stars", The Astrophysical Journal Supplement, Volume 208, Issue 1, 2013

3. Chanover et al, "Results from the Science Instrument Definition Team for the Gondola for High Altitude Planetary Science Project", American Astronomical Society, DPS meeting \#48, id.123.31, 2016

4. Esplin R. et al, "SABER Instrument Overview", Proc. SPIE Vol. 2268, p. 207-217, 1994

5. Vollmerhausen, Richard H., et al "Night illumination in the near-IR and short-wave infrared spectral bands and the potential for silicon and indium-gallium-arsenide imagers to perform night targeting”, SPIE Optical Engineering, Vol. 52, id $043202(2013)$

6. Noll S., et al, "An atmospheric radiation model for Cerro Paranal”, Astronomy \& Astrophysics, Volume 543, id.A92, $23 \mathrm{pp}, 2012$

7. Ledrew, Glenn (February 2001). "The Real Starry Sky", Journal of the Royal Astronomical Society of Canada. 95: 32., Febuary 2001

8. https://en.wikipedia.org/wiki/Stellar_classification

9. Houdashelt, M. L.; Bell, R. A.; Sweigart, A. V., "Improved Color-Temperature Relations and Bolometric Corrections for Cool Stars", The Astronomical Journal, Volume 119, Issue 3, pp. 1448-1469, 2000

10. Liebe, Carl C., "Accuracy Performance of Star Trackers - A Tutorial", IEEE Transactions On Aerospace and Electronic Systems Vol. 38, No. 2, April 2002

11. Dankanich John W., et al, "Planetary Balloon-Based Science Platform Evaluation and Program Implementation - Final Report", NASA/TM 2016-218870, 2016

12. Jim, K., ; Gibson, B.; Pier, E., "Daytime Sky Brightness Modeling of Haleakala along the GEO Belt", Proceedings of the Advanced Maui Optical and Space Surveillance Technologies Conference, 2012, id 68

13. Feruson, H., et al., "Image Quality Guidelines, NGST Monograph No. 7", Space Telescope Science Institute, 2001

14. Vollath, D., "Automatic Focusing by Correlated Methods", J. Microsc., 147, p279-288, 1987 